\title{
REVIEW
}

\section{The role of Fas-mediated apoptosis in thyroid disease}

\author{
M Andrikoula and A Tsatsoulis \\ Division of Endocrinology, Department of Medicine, University of Ioannina Medical School, Ioannina 45110, Greece \\ (Correspondence should be addressed to A Tsatsoulis; Email: atsatsou@cc.uoi.gr)
}

\begin{abstract}
Recent evidence has emphasized the importance of apoptosis in the maintenance of tissue homeostasis and the pathogenesis of malignant and immune diseases. Autoimmune thyroid diseases such as Hashimoto's thyroiditis and Graves' disease, as well as other autoimmune endocrine diseases, have been associated with dysregulation of apoptotic signaling pathways. In particular, dysfunction of the Fas apoptotic pathway or production of soluble factors including soluble Fas and soluble Fas ligand may be involved in the pathogenesis of these disorders. On the other hand, malignant thyroid cells may avoid Fas-mediated suicide possibly by expression of inhibitors of apoptosis and evade the immune system by inducing apoptosis on infiltrating lymphocytes. The delicate balance between cell proliferation and cell death through the Fas pathway may also play an important role in the control of thyroid cell mass and goitrogenesis. This review analyzes the current evidence on the role of Fasmediated apoptosis in the pathogenesis of thyroid diseases including Hashimoto's thyroiditis, Graves' disease, thyroid cancer and goiter. However, the exact mechanisms involved in the regulation of apoptosis in thyroid disease remain unclear. Further investigation is needed.
\end{abstract}

European Journal of Endocrinology 144 561-568

\section{Introduction}

The term 'apoptosis' was introduced into modern scientific writing by Kerr et al. in 1972 (1) to describe the special morphology of physiological cell death. The word itself is an ancient Greek word meaning the 'falling off' of leaves from a tree or petals from a flower and was originally used in the medical and philosophical writings of classical Greek and Roman times (2).

Apoptosis or 'programmed cell death' represents the regulated activation of a pre-existing death program encoded in the genome. It is a highly orchestrated form of cell death and plays a central role in the control of tissue cell numbers during development and homeostasis together with important functions, such as cell proliferation and differentiation (3-5). Dysregulation of apoptosis, on the other hand, may be involved in the pathogenesis of human disease (6). To date, diseases of the endocrine system such as Hashimoto's thyroiditis (HT), Graves' disease (GD) and diabetes mellitus type 1 have been considered to be autoimmune in origin. Recent evidence suggests that programmed cell death is also involved in the pathogenesis of these disorders. The Fas pathway has been demonstrated to be an important apoptotic pathway in the thyroid, but its role in the pathogenesis of thyroid diseases is controversial and a subject of much debate. This review summarizes the available evidence on the role of Fas-mediated apoptosis in thyroid diseases such as autoimmune thyroiditis, GD, thyroid cancer and goiter. An understanding of the function and regulation of the Fas pathway in the thyroid may provide insights into potential pathogenic mechanisms and may enable the design of more effective strategies for prevention and treatment of thyroid diseases.

\section{Apoptosis induction by Fas}

There are two major pathways leading to apoptosis: (a) positive induction by ligand binding to a plasma membrane receptor and (b) negative induction by loss of a trophic signal. The apoptotic mechanism of a cell involves the death receptors, the adaptor molecules, the caspase cascade, the mitochondria and the Bcl-2 proteins $(7,8)$.

The death receptors known today belong to the tumor necrosis factor receptor (TNFR) gene superfamily which is defined by similar, cystein-rich extra cellular domains. The death receptors form a subgroup of the superfamily TNFR, containing a homologous cytoplasmic sequence of 80 amino acids termed the 'death domain' $(9,10)$. The death domain (DD) bridges the death receptors with the apoptotic machinery, but in some cases they mediate functions different from apoptosis, such as the activation of the nuclear factor $\kappa \mathrm{B}$ (NK-кB). Some adaptor molecules transmitting 
apoptotic signals from death receptors contain DDs themselves.

One of the best characterized death receptors is CD95/Fas (Fig. 1). As soon as it is activated by its ligand, Fas ligand/CD95L, the apoptotic signal is transmitted through the adaptor molecule Fas-associated DD (FADD)/Mort1 to the zymogen form of cysteinyl-aspartate-specific protease (caspase)-8 termed FLICE (FADD-like interleukin-1 $\beta$ converting enzyme) (11). Upon recruitment by FADD, caspase- 8 oligomerization leads to its activation through self-cleavage (12). Subsequently, caspase-8 activates downstream effector caspases, such as caspase-9, causing an apoptotic demise of the cell. Studies with mice lacking

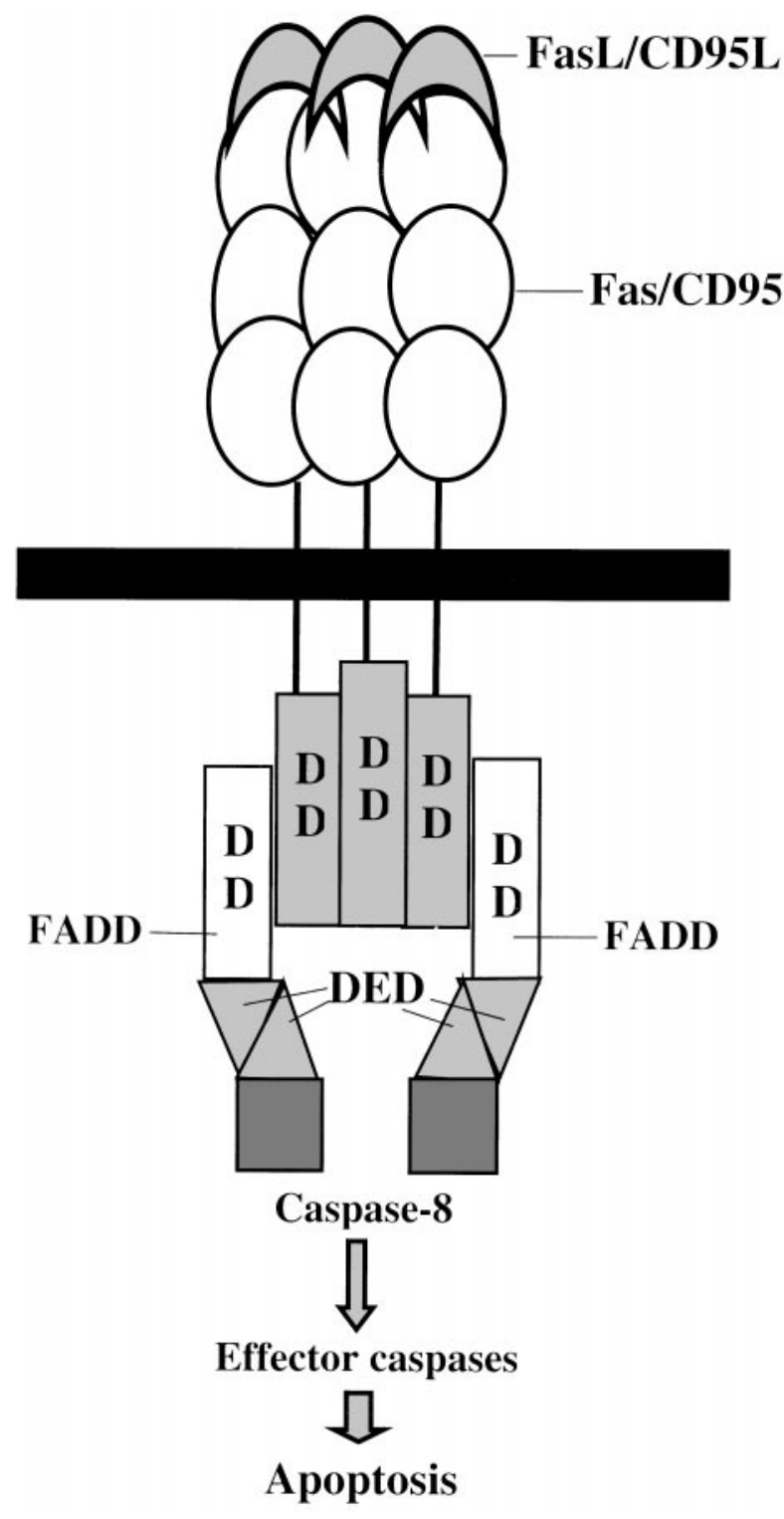

Figure 1 The death receptor Fas and apoptosis induction by Fas-FasL showing the death domains (DD) and the death effector domains (DED). the FADD gene indicated that this adaptor molecule is essential for apoptosis induction by Fas (13). The activation of caspases plays a pivotal role in the initiation and execution of apoptosis induced by various stimuli. To date, 14 different members of caspases have been identified in mammalian cells.

The gene responsible for Fas protein is located on human chromosome 10. The Fas antigen is a 335 amino acid, type I cell surface protein belonging to the TNFR family $(14,15)$. FasL is a type II transmembrane protein of the same family, which has the ability to bind to Fas (16). The induction of the apoptotic mechanism requires Fas activation either by anti-Fas antibody or by FasL. Like other TNF family members, FasL is a homotrimeric molecule, which means that each FasL trimer binds three Fas molecules (10).

It is already known that Fas and FasL play an important role in three types of physiologic apoptosis: (1) peripheral deletion of activated mature T cells at the end of an immune response, (2) killing of targets such as various infected cells or cancer cells by cytotoxic $\mathrm{T}$ cells and by natural killer cells (Fig. 2), and (3) killing of inflammatory cells at 'immune-privileged' sites such as the eye and the testis $(16,17)$.

Recent studies indicate that apoptosis induced by Fas-FasL is implicated in the pathogenesis of autoimmune endocrine diseases, such as GD, HT, diabetes mellitus type 1 and Addison's disease.

\section{The role of the Fas pathway in immune-mediated apoptosis}

The central pathogenic event in the development of autoimmune endocrine diseases is the immunemediated destruction of endocrine cells. HT is caused by the cytotoxicity of thyroid follicular cells (18). A similar mechanism is considered to lead to Addison's disease, where cytotoxicity of adrenal cortical cells results in adrenal insufficiency (19), and type 1 diabetes mellitus where cytotoxicity of islet $\beta$ cells results in inadequate insulin secretion and hyperglycemia (20). Islet $\beta$ cells were found to be destroyed by apoptosis in both animal models of diabetes and in humans with type 1 diabetes. However, when $\beta$ cell cytotoxicity mediated by Fas was prevented, the animals did not develop diabetes (21). Thus, apoptosis induced by Fas is probably involved in the pathogenesis of autoimmune diseases of the endocrine glands, including the thyroid.

Immunohistochemical studies of thyroid tissue sections revealed an increased number of apoptotic follicular cells in HT $(22-24)$, most of them on the periphery of infiltrating lymphocytes. Conversely, the number of apoptotic cells is decreased in GD where the lymphocytic infiltration is milder. The above findings suggest that the apoptotic destruction of thyroid tissue is mediated by the immune system with mechanisms that remain unclear. 


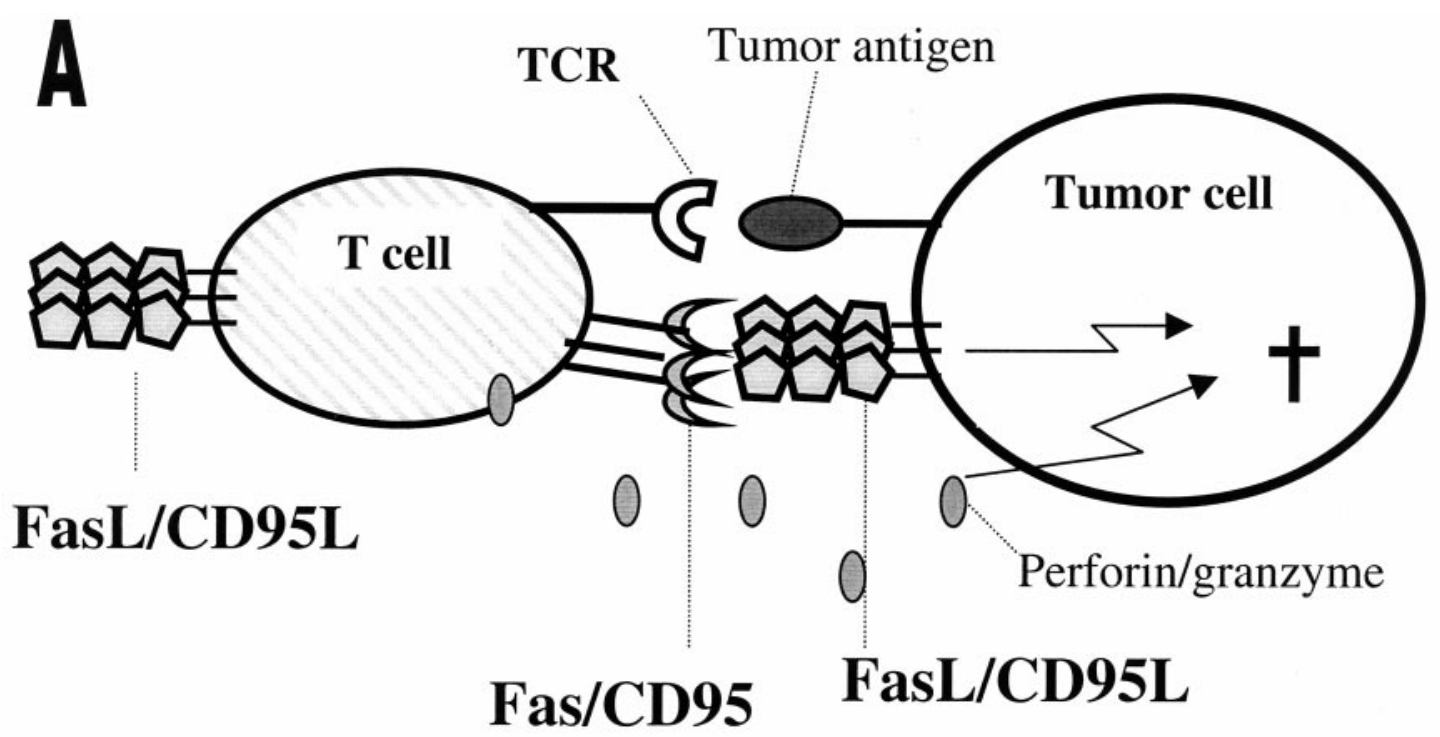

B

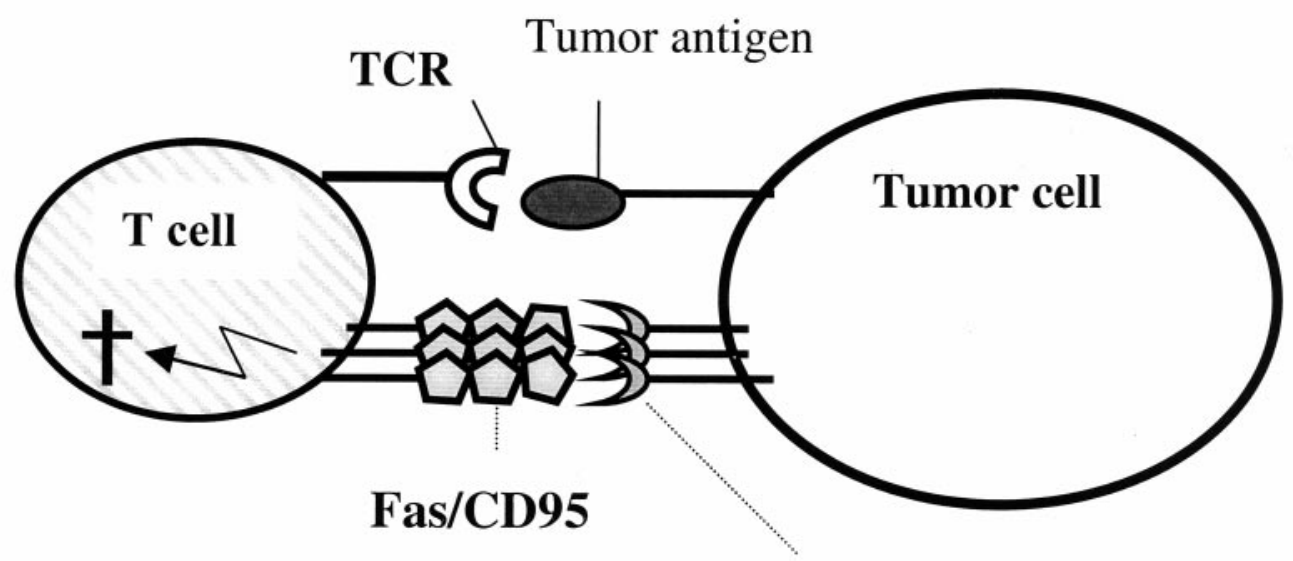

FasL/CD95L

Figure $2 \mathrm{~T}$ cell interactions with tumor cells. (A) The T cell recognizes tumor cells via the T cell receptor (TCR) and kills them by Fas-FasL interaction or by releasing perforin/granzyme. (B) Tumor cells are resistant to apoptosis and express FasL. The T cell recognizes tumor cells but undergoes apoptosis by Fas-FasL interaction (tumor counterattack).

According to recent evidence, CD8 cells cause cellular cytotoxicity in target cells not by necrosis or inflammatory destruction of the cells, but through the induction of apoptosis (25). Apoptosis is induced by triggering of membrane receptors of the target cells either by ligands on the lymphocytes or soluble factors that are released and delivered to the target cell (26).

Fas ligand expression is increased on activated natural killer cells, resulting in an increased cytotoxicity potential of these cells. Tumor cells often escape immune surveillance by down-regulation of Fas antigen or by constitutive expression of FasL (27).

Therefore, the Fas pathway plays a central role in immune-mediated apoptosis and the regulation of immune responses. This is the reason why recent studies of the pathogenesis of autoimmune thyroid disease have focused on this death pathway and its regulatory function in the thyroid gland. 


\section{Fas ligand expression in the thyroid}

Fas ligand expression has been reported to occur only in activated cytotoxic $\mathrm{T}$ lymphocytes and natural killer cells and tissues such as the eye and testis, which are regarded as 'immune privileged' $(17,28)$. Cells from these tissues are protected from cytotoxic T cells by FasL expression which binds to Fas antigen on lymphocytes and induces apoptosis. Recently, FasL expression was also detected in some tumor cells including those of epithelial origin (27). By expressing FasL, these cells are able to induce apoptosis in Fas-positive cells of the immune system, escaping immune surveillance. FasL expression on thyrocytes is controversial. Giordano et al. (29) reported constitutive FasL expression on thyroid cells from both non-toxic goiter and HT tissues. Fas and FasL expression on thyrocytes suggests that cell death may be spontaneously induced in neighboring thyroid cells (Fig. 3). Indeed, cultured normal thyrocytes expressed Fas antigen after treatment with interleukin $1 \beta$ (IL-1 $\beta)$ and underwent apoptosis by Fas-FasL interaction (29). However, massive apoptosis has not been reported in vivo. In contrast, apoptosis and destruction of thyroid cells appear to occur more gradually (22-24), questioning the relevance of this theory (30).

The report by Giordano et al. (29) was further criticized because it employed antibodies that appeared to recognize a protein that was not FasL (31). Furthermore, other groups examined the expression of FasL on thyrocytes using different techniques and failed to show the expression of mRNA for FasL in thyrocytes from normal, HT and GD thyroid tissues (32, 33). A more recent study (34) employing monoclonal antibodies against human FasL reported that FasL expression was only observed in GD thyroid tissues and not in normal thyroids. Other investigators have examined the expression of FasL in thyroid carcinomas

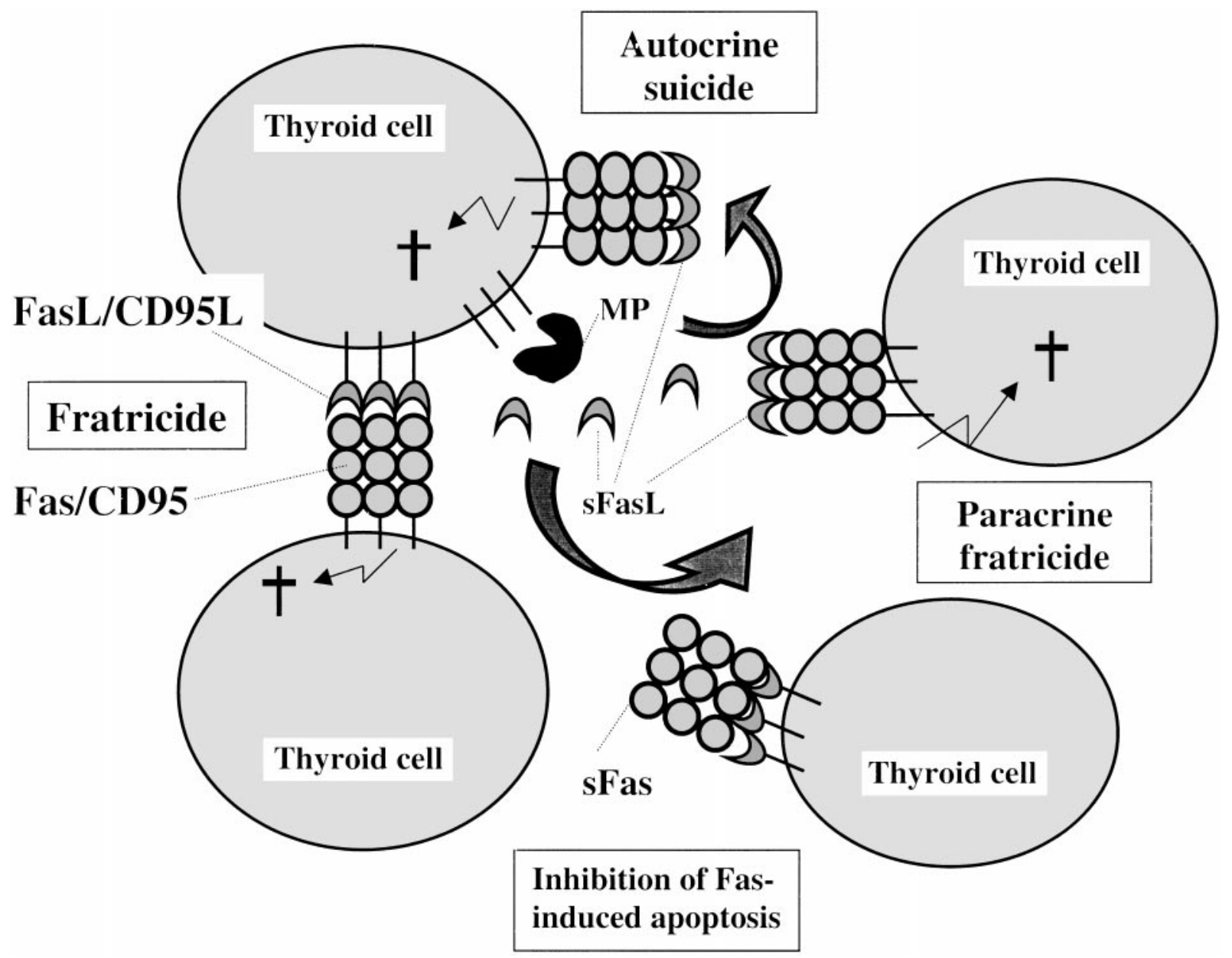

Figure $3 \mathrm{~A}$ model for thyroid cell destruction. Neighboring thyroid cells possibly destroy each other by expressing Fas and FasL. The soluble form of FasL (sFas), resulting from cleavage of Fas by a metalloproteinase (MP), can induce apoptosis in the same or adjacent cells (autocrine suicide or paracrine fratricide). 
(35). They also showed that FasL was expressed only in thyroid carcinomas, not in normal thyroid cells, and that it varied in the different types of cancer.

The apparent discrepancies in FasL expression in thyroid tissue among the different studies may be due to differences in methods or patient selection with regard to disease activity and degree of lymphocytic infiltration. However, the weight of evidence suggests that constitutive expression of FasL does not occur in normal thyrocytes but thyroid cells in certain thyroid diseases may express functional FasL. The trigger for this expression is not yet defined.

\section{Soluble form of Fas and FasL and apoptosis of thyroid cells}

Recently, Cheng et al. (36) described an alternatively spliced Fas mRNA that results from deletion of exons 3, 4, 6 and 7. This mRNA encodes the soluble form of Fas (sFas) lacking the transmembrane domain of this receptor. sFas is supposed to inhibit Fas-FasL interaction and protect target cells from Fas-induced apoptosis. Serum levels of sFas were reported to be elevated in patients with systemic lupus erythematosus or rheumatoid arthritis (37). Some studies showed a significant correlation between sFas levels and the clinical and laboratory findings of these diseases, although other groups were not able to confirm this suggestion.

The serum levels of sFas have recently been measured in patients with autoimmune thyroid diseases (38). In contrast to normal controls, sFas was increased in thyrotoxic patients with GD, but reduced in patients with GD in remission. Additionally, sFas was normal in hypothyroid patients with HT and thyrotoxic patients with silent thyroiditis. The above data were later confirmed by others (39), who also measured high levels of sFas in thyrotoxic patients with GD. The levels of sFas were reduced when patients became euthyroid after treatment with antithyroid drugs for $6-8$ weeks. It is not yet clear if the elevated serum levels of sFas result from either the increased production of sFas in thyroid cells and infiltrating lymphocytes, or the increased number of these cells. Although the induction of sFas production by inflammatory cytokines seems to support the former theory, the latter is also possible as thyroid hyperplasia is a characteristic of GD.

Increased sFas in GD indicates increased expression of a Fas mRNA variant (which results from alternative splicing) and reduced Fas expression on cell surface. The increased sFas is likely to promote thyrocyte proliferation and production of anti-TSH receptor antibody by protecting thyroid cells and autoreactive $\mathrm{B}$ cells respectively from apoptosis.

FasL is also detected in a soluble form (sFasL) resulting from cleavage at the cell membrane by a metalloproteinase (40). Similar to soluble TNF, sFasL can induce programmed cell death on Fas antigen-expressing target cells (Fig. 3).

\section{Regulation of the Fas pathway in the thyroid gland}

Immunohistochemical studies of thyroid tissue sections found Fas protein present on thyroid follicular cells from healthy individuals, as well as from patients with thyroid carcinomas, GD and HT, although the extent and specific disorders associated with this expression differed between the studies $(22,23)$. In thyrocyte cultures, Fas protein was detected either by antibodies against Fas antigen $(24,41,42)$, or by Western blots in cell lysates (41). However, it was recently reported that Fas expression is induced on thyrocytes only under the influence of inflammatory cytokines, such as interferon- $\gamma($ IFN- $\gamma$ ) or IL-1 $\beta$ (29). The differences between the studies may result from differences in reagents or tissues examined.

The presence of Fas alone, however, may not be enough to induce apoptosis in cells. Recent evidence suggests that regulation of Fas-induced apoptosis in thyroid cells may be more complex than previously thought. Indeed, studies by Arscott et al. (41) suggest that the Fas pathway is blocked at some point by a short-lived (labile) inhibitor. It was reported that the induction of apoptosis on thyrocytes using anti-Fas monoclonal antibody requires the presence of cycloheximide (CHX), which is an inhibitor of protein translation and blocks labile inhibitors of the Fas pathway. CHX was required even after treatment of thyroid cells with IFN- $\gamma$ or IL-1 $\beta$. Kawakami et al. (42) suggested that thyrotropin (TSH) also inhibits Fas expression and apoptosis on thyrocytes in vitro but this has not been shown by other investigators (41). The presence and regulation of such inhibitors of the Fas pathway may explain the susceptibility to different levels of apoptosis in the thyroid gland and may protect thyrocytes from Fas-induced apoptosis.

Recently, various inhibitors of the Fas pathway have been indentified, such as Fas-associated phosphatase (FAP-1) and inhibitors of apoptosis proteins (IAPs). FAP-1 is a protein tyrosine phosphatase that interacts with the carboxyl end of Fas, preventing signal transduction to FADD (43). IAPs form a family of viral inhibitor proteins that bind to the DD of FADD, preventing its interaction with FLICE and consequently the activation of caspases (44). This protein, which is called FLIP or FLICE-inhibitory protein (I-FLICE), inhibits apoptosis induced by Fas or other death receptors. Such inhibitors of the Fas pathway may contribute to the prevention of apoptosis in thyrocytes but their role has not been investigated in thyroid disease.

The anti-apoptotic protein $\mathrm{Bcl}-2$ is not believed to inhibit apoptosis induced by the Fas pathway but it may block apoptosis due to radiation, DNA damage or 
drugs (45). Indeed, differences in Bcl-2 expression have been reported in different thyroid conditions, indicating that regulation of this protein may also play a role in thyroid diseases $(24,46)$.

\section{Role of Fas pathway in the pathogenesis of thyroid diseases}

\section{Hashimoto's thyroiditis}

HT is characterized by an immune response to thyroid antigens and an intense lymphocytic infiltration of the thyroid gland, resulting in the destruction of thyroid follicular cells and clinical hypothyroidism (47). HT is associated with thyroid-specific B cells as well as with $\mathrm{T}$ cell immune responses. There are antibodies to thyroid peroxidase and thyroglobulin, while the majority of intrathyroidal $\mathrm{T}$ cells are $\mathrm{CD} 8$ phenotype indicating cytotoxic activity against thyrocytes $(48,49)$. The above evidence is the reason why, until now, most of the investigations into the pathogenesis of autoimmune thyroid disease have focused on the immune system.

Extensive research in this field demonstrated that even the development of an antithyroid immune response does not necessarily lead to thyroid cell destruction and clinical hypothyroidism. This is the reason why other factors have been examined for their contribution to the pathogenesis of autoimmune thyroid disease (30). As mentioned above, thyroid follicular cells in tissue samples from HT exhibited strong staining for Fas and FasL and high apoptotic rate $(30.3 \%)$, while normal control follicles exhibited moderate Fas, minimal or no FasL and a low percentage of apoptosis (24). Immunostaining for Bcl-2 was weak in patients' thyroid follicles and high in normal controls. Infiltrating lymphocytes exhibited weak staining for FasL and strong for Bcl-2 $(24,46)$. These data suggest that, in HT, thyroid follicular cells undergo apoptosis by up-regulation of Fas and FasL and down-regulation of $\mathrm{Bcl}-2$ protein. Infiltrating lymphocytes do not seem to be directly involved in this process with their own FasL but they are likely to provide the appropriate cytokine milieu that results in the up-regulation of Fas and FasL and subsequently the activation of the apoptotic machinery.

\section{Graves' disease}

GD is considered to be an autoimmune thyroid disorder. It is characterized by hyperplasia of thyrocytes resulting from stimulation with anti-TSH receptor autoantibodies. Although thyrocytes from GD patients express both Fas and FasL in vivo, apoptosis was only occasionally found in the GD thyroids (34). The expression of FasL in GD thyrocytes, the downregulation of Fas expression by the stimulation of TSH receptor and the over expression of Bcl-2 (an inhibitor of apoptosis) or the presence of a labile protein inhibitor (41) have been proposed as possible factors for the inhibition of Fas-mediated apoptosis in Graves' thyrocytes.

Other studies have addressed the role of sFas, which suppresses Fas-mediated apoptosis in the pathogenesis of GD. They confirmed that serum sFas increases in GD and provided evidence of local production of sFas by thyrocytes and its regulation by cytokines. These studies suggest that sFas, by interfering with the Fas-FasL interaction, may also play a role in the pathogenesis of GD $(38,39)$.

Apoptosis induced by Fas may also be involved in the peripheral deletion of B cells activated by self antigens. The increased sFas may block this process and B cells escaping that deletion may be responsible for the production of TSH receptor antibody in GD (38). Apparently, the soluble form of Fas seems to be involved in the pathogenesis of GD, but further studies are needed to determine either the pathogenic or the protective role of sFas in this disease.

The increased expression of FasL in thyrocytes from GD patients may help to maintain homeostasis in the thyroid by eliminating infiltrating lymphocytes and hyperplastic thyrocytes by Fas-mediated apoptosis (34). Further studies are required to elucidate the mechanisms responsible for the FasL expression in the thyroid in GD.

\section{Thyroid carcinomas}

Apoptosis may play an important role in cancer. Many tumors have been found to be not only resistant to killing by apoptosis, but also to express functional FasL constitutively. This suggests that they may carry 'weapons', like the death ligands, to fight the immune system. The result of this 'tumor counterattack' may be the depletion of the attacking anti-tumor lymphocytes by the tumor, immunosuppression and immune escape. The ability of the neoplastic cell to escape the host's immune surveillance and to kill infiltrating immune cells is a strong survival advantage during the course of cancer progression (35).

In contrast to normal thyroid tissue, papillary carcinomas showed FasL immunoreactivity, especially in areas of poor and/or squamous differentiation. In a co-culture experiment, thyroid carcinoma cells were found to induce FasL-dependent apoptosis in Fassensitive cells of lymphocytic origin, suggesting that FasL in thyroid carcinomas is functional. From the laboratory findings, it appears that FasL expression is limited to papillary, follicular and Hurthle carcinomas, while FasL immunoreactivity in medullary carcinomas was poor or absent (35).

Consequently, it has been suggested that FasL expression helps thyroid carcinoma cells to evade the immune system by inducing apoptosis of infiltrating lymphocytes. This raises the question of why FasLexpressing tumor cells do not undergo apoptotic 
suicide/fratricide as they express Fas receptor. Previous studies showed a low rate of apoptotic cells in thyroid carcinomas. It appears that FasL expressed on the neoplastic thyrocyte is directed against cells of the immune system and not the thyrocyte itself, probably due to a defect in the tumor cell apoptotic pathway, or the presence of a protein inhibitor of apoptosis that protects the cell from suicide.

\section{Goiter}

Under normal conditions, the size of the thyroid gland during adulthood remains relatively constant because cell proliferation is balanced by cell death. This balance may be disturbed in human goiter, which is the result of diffuse or focal hyperplasia of thyroid follicular cells at one or multiple sites within the thyroid gland.

The role of apoptosis and the involvement of the Fas pathway were previously investigated in rat models of goiter during the stages of goiter formation and involution (50). Rats were fed a low iodine diet and a goitrogen, 6-propyl-2-thiouracil. Later, the same rats with goiter were fed a high iodine diet to study the phase of involution. The major findings of this study were an increased number of apoptotic cells paralleled by an increased number of Fas-positive cells during the development of goiter and the early stage of involution but a decreased number of Fas-positive cells in the late stage of goiter involution. Constitutive expression of FasL was observed throughout the experiment. These findings suggest that Fas expression may serve as a limiting factor for the induction of Fas-mediated apoptosis.

We have studied the expression of Fas in vivo in thyrocytes from patients with non-toxic nodular goiter who underwent fine needle aspiration biopsy for diagnostic reasons (authors' unpublished observations). The results showed that the expression of Fas protein is low in human nodular goiter, indicating reduced thyroid cell apoptosis through the Fas pathway. Therefore, the regulation of Fas protein expression may play a role in the pathogenesis of goiter by upsetting the normal balance between thyroid cell proliferation and apoptosis in favor of the former process.

Besides the Fas/FasL system, iodine may also be involved in the death of thyrocytes and the control of thyroid gland mass. High doses of iodine inhibit the proliferation of FRTL-5 cells and induce cell toxicity both in vivo and in vitro, possibly due to an excessive production of free radicals. The role of iodine in inducing apoptosis was also investigated in thyroid cell cultures. Low concentrations of iodine were capable of inhibiting apoptosis, while high concentrations resulted in increased Fas-induced apoptosis (51).

The above findings indicate that the delicate balance between thyroid cell proliferation and apoptosis may play a central role in the control of thyroid gland mass. Further studies are needed to clarify the role of apoptosis and the Fas pathway in the pathogenesis of goiter.

\section{Conclusion}

Fas-mediated apoptosis may play an important role in thyroid tissue homeostasis as well as in thyroid disease, since morphological and biochemical features of programmed cell death have been observed in normal thyroid glands and in various thyroid diseases. However, the mechanisms and regulation of apoptosis in thyrocytes remain unclear. Further studies are required to clarify the signaling components of programmed cell death present in thyroid cells and the in vivo regulatory mechanisms of this process. The regulation of apoptotic cell death pathways in the thyroid may alter the expression of thyroid diseases by modifying the susceptibility of thyroid cells to Fas-mediated apoptosis and may offer new therapeutic options.

\section{References}

1 Kerr JF, Wyllie AH \& Currie AR. Apoptosis: a basic biological phenomenon with wide-ranging implications in tissue kinetics. British Journal of Cancer 197226 239-257.

2 Esposti MD. Apoptosis: who was first? Cell Death and Differentiation 19985719.

3 Sen S. Programmed cell death: concept, mechanism and control. Biological Reviews of the Cambridge Philosophical Society 199267 287-319.

4 Barinaga M. Forging a path to cell death. Science 1996273 735-737.

5 Ameisen JC. The origin of programmed cell death. Science 1996 272 1278-1279.

6 Thompson CB. Apoptosis in the pathogenesis and treatment of disease. Science 1995267 1456-1462.

7 Mountz JD, Zhang H-G, Hsu H-C, Fleck M, Wu J, Al-Maini MH et al. Apoptosis and cell death in the endocrine system. Recent Progress in Hormone Research 199954 235-269.

8 Steller H. Mechanisms and genes of cellular suicide. Science 1995 267 1445-1449.

9 Tartaglia L, Ayres TM, Wong GH \& Goeddel DV. A novel domain within the $55 \mathrm{kd}$ TNF receptor signals cell death. Cell $1993 \mathbf{7 4}$ 845-853.

10 Ashkenazi A \& Dixit VM. Death receptors: signaling and modulation. Science 1998281 1305-1308.

11 Boldin M, Goncharov T, Goltsev YV \& Wallach D. Involvement of MACH, a novel MORT1/FADD-interacting protease, in Fas/APO1 and TNF receptor-induced cell death. Cell $1996 \mathbf{8 5} 803-815$.

12 Muzio M, Stockwell BR, Stennicke HR, Salvesen GS \& Dixit VM. An induced proximity model for caspase-8 activation. Journal of Biological Chemistry $19982732926-2930$.

13 Zhang J, Cado D, Chen A, Kabra NH \& Winoto A. Fas-mediated apoptosis and activation-induced T-cell proliferation are defective in mice lacking FADD/Mort1. Nature 1998392 296-300.

14 Itoh N, Yonehara S, Ishii A, Yonehara M, Mizushima S, Sameshima $\mathrm{M}$ et al. The polypeptide encoded by the cDNA for human cell surface antigen Fas can mediate apoposis. Cell 1991 66 233-243.

15 Nagata S \& Golstein P. The Fas death factor. Science 1995267 1449-1456.

16 Suda T, Takahashi T, Golstein P \& Nagata S. Molecular cloning and expression of the Fas ligand, a novel member of the tumor necrosis factor family. Cell 199375 1169-1178. 
17 Griffith TS, Brunner T, Fletcher SM, Green DR \& Ferguson TA. Fas ligand-induced apoptosis as a mechanism of immune privilege. Science 1995270 1189-1192.

18 Weetman AP \& McGregor AM. Autoimmune thyroid disease. Endocrine Reviews 19845 309-337.

19 Winqvist O, Soderbergh A \& Kampe O. The autoimmune basis of adrenocortical destruction in Addison's disease. Molecular Medicine Today 19962 282-289.

20 Foulis AK. The pathology of the endocrine pancreas in type 1(insulin-dependent) diabetes mellitus. APMIS $1996 \mathbf{1 0 4}$ 161-167.

21 Chervonsky AV, Wang Y, Wong F, Visintin I, Flavell RA, Janeway CA Jr et al. The role of Fas in autoimmune diabetes. Cell 199789 17-24.

22 Tanimoto C, Hirakawa S, Kawasaki H, Hayakawa N \& Ota Z. Apoptosis in thyroid diseases: a histochemical study. Endocrine Journal 199542 193-201.

23 Kotani T, Aratake Y, Hirai K, Fukazawa Y, Sato H \& Ohtaki S. Apoptosis in thyroid tissue from patients with Hashimoto's thyroiditis. Autoimmunity 199520 231-236.

24 Hammond LJ, Lowdell MW, Cerrano PG, Goode AW, Bottazzo GF \& Mirakian R. Analysis of apoptosis in relation to tissue destruction associated with Hashimoto's autoimmune thyroiditis. Journal of Pathology $1997 \mathbf{1 8 2} 138-144$.

25 Ridgway WM, Weiner HL \& Fathman CG. Regulation of autoimmune response. Current Opinions in Immunology 19946 946-955.

26 Lowin B, Hahne M, Mattmann C \& Tschopp J. Cytolytic T-cell cytotoxicity is mediated through perforin and Fas lytic pathways. Nature 1994370 650-652.

27 Hahne M, Rimoldi D \& Schroter M. Melanoma cell expression of Fas (Apo-1/CD95) ligand: implications for tumor immune escape. Science $1996 \mathbf{2 7 4} 1363-1366$.

28 Bellgrau D, Gold D, Selawry H, Moore J, Franzusoff A \& Duke RC. A role for CD95 ligand in preventing graft rejection. Nature 1995 377 630-632.

29 Giordano C, Stassi G, DeMaria R, Todaro M, Richiusa P, Papoff G et al. Potential involvement of Fas and its ligand in the pathogenesis of Hashimoto's thyroiditis. Science $1997 \mathbf{2 7 5}$ 960-963.

30 Arscott PL \& Baker JR Jr. Apoptosis and thyroiditis. Clinical Immunology and Immunopathology 199887 207-217.

31 Fiedler P, Schaetzlein CE \& Eibel H. Constitutive expression of FasL and thyrocytes. Science 19982792015.

32 Rymaszewski M, Arscott PL, Knapp J, Bretz JD, Thompson NW, Bartron $\mathrm{J}$ et al. An examination of Fas ligand expression on thyrocytes (Meeting Abstract). Thyroid 19977149.

33 Stokes TA, Rymaszeuski M, Arscott PL, Wang SJ, Bretz JD, Bartron J et al. Constitutive expression of FasL in thyrocytes (Technical comments). Science 1998279 2015a.

34 Hiromatsu Y, Hoshino T, Yagita H, Koga M, Sakisaka S, Honda J et al. Functional Fas ligand expression in thyrocytes from patients with Graves' disease. Journal of Clinical Endocrinology and Metabolism $1999842896-2902$.

35 Mitsiades N, Poulaki V, Mastorakos G, Tseleni-Balafouta S, Kotoula V \& Koutras DA. Fas ligand expression in thyroid carcinomas: a potential mechanism of immune evasion. Journal of Clinical Endocrinology and Metabolism $1999 \mathbf{8 4}$ 2924-2932.

36 Cheng J, Zhou T, Liu C, Shapiro JP, Brauer MJ, Kiefer MC et al. Protection from Fas-mediated apoptosis by a soluble form of the Fas molecule. Science 1994263 1759-1762.
37 Jodo S, Kobayashi S, Kayagaki N, Ogura N, Feng Y, Amasaki Y et al. Serum levels of soluble Fas/APO-1 (CD95) and its molecular structure in systemic Lupus erythematosus (SLE) and other autoimmune diseases. Clinical and Experimental Immunology $199710789-95$.

38 Shimaoka Y, Hidaka Y, Okumura M, Takeoka K, Tada H \& Amino N. Serum concentration of soluble Fas in patients with autoimmune thyroid diseases. Thyroid 1998 8 43-47.

39 Hiromatsu Y, Bednarczuk T, Soyejima E, Miyake I, Yang D, Fukazawa $\mathrm{H}$ et al. Increased serum soluble Fas in patients with Graves' disease. Thyroid 19999 341-345.

40 Tanaka M, Suda T, Takahashi T \& Nagata S. Expression of the functional soluble form of human Fas ligand in activated lymphocytes. EMBO Journal 199514 1129-1135.

41 Arscott PL, Knapp J, Rymaszewski M, Bartron JL, Bretz JD \& Thompson ND. Fas(APO-1, CD95)-mediated apoptosis in thyroid cells is regulated by a labile protein inhibitor. Endocrinology 1997 138 5019-5027.

42 Kawakami A, Eguchi K, Matsuoka N, Tsuboi M, Kawabe Y, Ishikawa $\mathrm{N}$ et al. Thyroid-stimulating hormone inhibits Fas antigen-mediated apoptosis of human thyrocytes in vitro. Endocrinology 1996137 3163-3169.

43 Yanagisawa J, Takahashi M, Kanki H, Yano-Yanagisawa H, Tazunoki T, Sawa E et al. The molecular interaction of Fas and FAP-1: a tripeptide blocker of human Fas interaction with FAP-1 promotes Fas-induced apoptosis. Journal of Biological Chemistry $19972728539-8545$.

$44 \mathrm{Hu} \mathrm{S}$, Vincenz C, Ni J, Gentz R \& Dixit VM. I-FLICE, a novel inhibitor of tumor necrosis factor receptor-1-and CD95induced apoptosis. Journal of Biological Chemistry $1997 \mathbf{2 7 2}$ 17255-17257.

45 Chiu UK, Walsh CM, Liu CC, Reed JC \& Clark WR. Bcl-2 blocks degranulation but not Fas-based cell mediated cytotoxicity. Journal of Immunology $19951542023-2032$.

46 Mitsiades N, Poulaki V, Kotoula V, Mastorakos G, TseleniBalafouta ST, Koutras DA et al. Fas/Fas ligand up-regulation and Bcl-2 down-regulation may be significant in the pathogenesis of Hashimoto's thyroiditis. Journal of Clinical Endocrinology and Metabolism 199883 2199-2203.

47 Weetman AP \& McGregor AM. Autoimmune thyroid disease: further developments in our understanding. Endocrine Reviews $199415788-830$.

48 Bermann M, Magee M \& Koenig RJ. Differential autoantibody responses to thyroid peroxidase in patients with Grave's disease and Hashimoto thyroiditis. Journal of Clinical Endocrinology and Metabolism 199377 1098-1101.

49 DeGroot LJ \& Quintans J. The causes of autoimmune thyroid disease. Endocrine Reviews 198910 537-562.

50 Tamura M, Kimura H, Koji T, Tominaga T, Ashizawa K, Kiriyama $\mathrm{T}$ et al. Role of apoptosis of thyrocytes in a rat model of goiter. A possible involvement of Fas system. Endocrinology $19981393646-3653$.

51 Felkamp J, Pascher E, Perniok A \& Scherbaum VA. Fas-mediated apoptosis is inhibited by TSH and iodine in moderate concentrations in primary human thryocytes in vitro. Hormone and Metabolic Research 199931 355-358.

Received 4 August 2000

Accepted 16 February 2001 